\title{
An Efficient Technique for Determining the Steady-State Membrane Potential Profile in Tissues with Multiple Cell Types
}

\author{
V Jacquemet, CS Henriquez \\ Duke University, Durham, NC, USA
}

\begin{abstract}
Most simulations of cardiac electrophysiology use the steady state as initial condition. Spatial variations in steady-state membrane potential may arise due to ischemia, coupling with fibroblasts, or local changes in intrinsic resting potential. In large scale models, simulating free evolution until the steady-state is reached may be computationally expensive when long time constants or slow concentration drifts are involved in the cell models.

This paper describes a dedicated Newton-based rootfinding solver to determine the steady state of a tissue in which two or more cell types coexist in the monodomain framework. This approach was applied to a $2 \mathrm{D} \mathrm{mi-}$ crostructural tissue model in which myocytes were coupled to fibroblasts, leading to an inhomogeneous elevation of the myocyte resting potential.
\end{abstract}

\section{Introduction}

Models of cardiac electrophysiology models simulating the propagation of the electrical impulse use in most cases the steady state as initial condition. In a tissue with a single cell type and uniform membrane properties, the resting state is necessarily uniform. As a result, it can be computed in a single cell model by letting this cell evolve from an arbitrary initial state until the steady state is reached. Even in this simple case, slow transient drifts in ionic concentrations make it difficult to establish that the steady state is indeed reached [1].

But the steady state is not always uniform. Spatial variations in steady-state membrane potential may arise due to ischemia, coupling with fibroblasts, or local changes in intrinsic resting potential. Since the sodium channels are very sensitive to the changes in resting potential, such variations can result in significant changes in excitability and conduction velocity. In large scale models, simulating free evolution until the steady-state is reached may be com- putationally expensive when long time constants or slow concentration drifts are involved in the cell models. For instance, the MacCannell et al. fibroblast model [2] has an intrinsic resting potential of $-49.4 \mathrm{mV}$ and one of its gating variables has a relaxation time in the range 1500 to $5300 \mathrm{~ms}$. If such fibroblast was coupled to myocyte, computing the steady state would require to simulate at least 5 to $10 \mathrm{~s}$.

In a previous work, a combination of analytical and numerical tools was used to identify the steady states in detailed atrial cell models [3]. This paper extends this approach to multi-cellular structures incorporating multiple cell types. The practical use of the resulting algorithm is illustrated through a one-dimensional model and a twodimensional microstructural tissue model, both incorporating myocytes and fibroblasts coupled through gap junctions.

\section{Methods}

\subsection{Problem statement}

In the framework of the monodomain approximation, the system of equations describing electrical propagation in a cardiac tissue reads [4]:

$$
\begin{aligned}
\beta C_{m} \frac{\partial V_{m}}{\partial t} & =\nabla \cdot \sigma \nabla V_{m}-\beta I_{\mathrm{ion}}\left(V_{m}, \mathbf{q}, \mathbf{x}\right) \\
\frac{\mathrm{d} \mathbf{q}}{\mathrm{d} t} & =\mathbf{F}\left(V_{m}, \mathbf{q}, \mathbf{x}\right)
\end{aligned}
$$

where $V_{m}$ is the membrane potential field, $\beta$ is the surfaceto-volume ratio, $C_{m}$ is the membrane capacitance per unit area, $\sigma$ is the effective conductivity, $I_{\text {ion }}$ is the ionic current across the membrane, $\mathbf{q}$ describes the internal state of the membrane (e.g. the channel gates), and $\mathbf{F}$ is associated with a membrane kinetics model. The explicit dependency in $\mathbf{x}$ of $I_{\text {ion }}$ and $\mathbf{F}$ indicates that the model parameters or even the formulation of the membrane kinetics may vary 
with space. This enables the introduction of multiple cell types.

The objective is to find the steady-state solution(s) of this system, that is, to find fields $V_{m}$ and $\mathbf{q}$ satisfying

$$
\begin{aligned}
\nabla \cdot \sigma \nabla V_{m} & =\beta I_{\text {ion }}\left(V_{m}, \mathbf{q}, \mathbf{x}\right) \\
\mathbf{F}\left(V_{m}, \mathbf{q}, \mathbf{x}\right) & =0 .
\end{aligned}
$$

Suppose that there exists a function $\mathbf{G}\left(V_{m}, \mathbf{x}\right)$, smooth in $V_{m}$, such that

$$
\mathbf{F}\left(V_{m}, \mathbf{G}\left(V_{m}, \mathbf{x}\right), \mathbf{x}\right)=0 .
$$

Then, Eq. (4) can be substituted into Eq. (3), leading to the equation

$$
\nabla \cdot \sigma \nabla V_{m}=\beta I\left(V_{m}, \mathbf{x}\right),
$$

where $I$ is defined by

$$
I\left(V_{m}, \mathbf{x}\right)=I_{\text {ion }}\left(V_{m}, \mathbf{G}\left(V_{m}, \mathbf{x}\right), \mathbf{x}\right) .
$$

The advantage is that the non-linear equation (6) to be solved involves only the field $V_{m}$. Subsection 2.2 will show how to compute the function $\mathbf{G}$ and subsection 2.3 will describe how Eq. (6) can be solved.

\subsection{Steady-state ionic current}

For most of the membrane kinetics models, the function $\mathbf{G}$ is well-defined and can be computed efficiently. For the sake of illustration, the case of the Luo-Rudy model [5] will be considered here. The details for more sophisticated models such as the Courtemanche et al. and the Nygren et al. atrial cell models can be found in [3].

In the Luo-Rudy model, $\mathbf{q}=\left(m, h, j, d, f, x_{1},\left[\mathrm{Ca}^{2+}\right]_{\mathrm{i}}\right)$, where the first 6 variables (gating variables) are governed by an equation of the form

$$
\frac{\mathrm{dy}}{\mathrm{d} t}=\frac{y_{\infty}\left(V_{m}\right)-y}{\tau_{y}\left(V_{m}\right)}
$$

with $y=m, h, j, d, f$ or $x_{1}$. The evolution of the intracellular calcium concentration $\left[\mathrm{Ca}^{2+}\right]_{\mathrm{i}}$ is described by

$$
\begin{aligned}
\frac{\mathrm{d}\left[\mathrm{Ca}^{2+}\right]_{\mathrm{i}}}{\mathrm{d} t} & =-\gamma I_{\mathrm{si}}+\frac{\left[\mathrm{Ca}^{2+}\right]_{0}-\left[\mathrm{Ca}^{2+}\right]_{\mathrm{i}}}{\tau} \\
I_{\mathrm{si}} & =g_{\mathrm{si}} d f\left(V_{m}-E_{\mathrm{si}}\right) \\
E_{\mathrm{si}} & =a-b \log \left[\mathrm{Ca}^{2+}\right]_{\mathrm{i}},
\end{aligned}
$$

where $\gamma,\left[\mathrm{Ca}^{2+}\right]_{0}, \tau, g_{\mathrm{si}}, a$ and $b$ are constant. For each gating variable $y$ at steady state, $y=y_{\infty}\left(V_{m}\right)$. In particular, $d$ and $f$ can be replaced by $d_{\infty}$ and $f_{\infty}$ in Eq. (10). In order to determine completely the function $\mathbf{G}$, we still have to solve Eq. (9) for $\left[\mathrm{Ca}^{2+}\right]_{i}$ at clamped $V_{m}$ when the time derivative vanishes. Algebraic manipulations lead to the formula:

$$
\left[\mathrm{Ca}^{2+}\right]_{\mathrm{i}}=W\left(c_{1} \exp c_{2}\right) / c_{1}
$$

where $W$ is the Lambert $\mathrm{W}$-function [6] and

$$
\begin{aligned}
& c_{1}=\left(g_{\mathrm{si}} d_{\infty} f_{\infty} \tau \gamma b\right)^{-1} \\
& c_{2}=c_{1}\left[\mathrm{Ca}^{2+}\right]_{0}-\left(V_{m}-a\right) / b .
\end{aligned}
$$

Therefore, the function $\mathbf{G}$ associated with the Luo-Rudy model (LR1) can be worked out analytically and is written as

$$
\begin{aligned}
\mathbf{G}_{\mathrm{LR} 1}\left(V_{m}\right)=( & m_{\infty}, h_{\infty}, j_{\infty}, d_{\infty}, \\
& \left.f_{\infty}, x_{1 \infty}, W\left(c_{1} \exp c_{2}\right) / c_{1}\right) .
\end{aligned}
$$

For each node $i$ whose membrane kinetics is described by the LR1 model, we set $I\left(V_{m}, \mathbf{x}_{i}\right)=$ $I_{\text {ion,LR } 1}\left(V_{m}, \mathbf{G}_{\mathrm{LR} 1}\left(V_{m}\right)\right)$, and so on for the other membrane kinetics models.

\subsection{Non-linear system solver}

Equation (6) will be assumed to be discretized in the following form using for instance the finite volume method:

$$
-\mathbf{M} \cdot \mathbf{V}=\mathbf{A} \cdot \mathbf{I}(\mathbf{V})
$$

where $\mathbf{V}$ is the membrane potential field (vector in $\mathrm{mV}$ ), $\mathbf{M}$ is the diffusion matrix (stiffness matrix in $\mathrm{mS}$, which is assumed to be symmetric non-negative definite), $\mathbf{A}$ is the diagonal matrix representing the area of membrane in each element (mass matrix in $\mathrm{cm}^{2}$ ), and $\mathbf{I}$ is the steadystate ionic current (vector in $\mu \mathrm{A} / \mathrm{cm}^{2}$ ) defined by Eq. (7).

Equation (16) can be solved iteratively using the Newton's method. The initial condition $\mathbf{V}^{0}$ is given by the cells being in their resting state as if they were uncoupled. Then, at step $n+1$, the update is obtained by solving the system:

$$
\mathbf{J}^{n} \cdot\left(\mathbf{V}^{n+1}-\mathbf{V}^{n}\right)=-\mathbf{M} \cdot \mathbf{V}^{n}-\mathbf{A} \cdot \mathbf{I}\left(\mathbf{V}^{n}\right),
$$

where the Jacobian matrix is given by

$$
\mathbf{J}^{n}=\mathbf{M}+\mathbf{A} \cdot \mathbf{I}^{\prime}\left(\mathbf{V}^{n}\right) .
$$

The $i$-th component of the vector $\mathbf{I}^{\prime}$ contains the membrane dynamic conductance $\partial I\left(V_{m}, \mathbf{x}_{i}\right) / \partial V_{m}$ computed at position $\mathbf{x}_{i}$. When a relatively small number of different cell types or membrane parameter sets are present in the tissue, the functions $I\left(V_{m}\right)$ and $\partial I\left(V_{m}\right) / \partial V_{m}$ can be precomputed and tabulated for each cell type in order to increase computational efficiency. The iterations are stopped when $\left\|\mathbf{V}^{n+1}-\mathbf{V}^{n}\right\|_{\infty}<\epsilon$, where typically $\epsilon=10^{-6} \mathrm{mV}$.

In the special case where the membrane response is linear, i.e. when $\mathbf{I}(\mathbf{V})=\mathbf{G}_{m}\left(\mathbf{V}-\mathbf{V}^{0}\right)$ with $\mathbf{G}_{m}$ being a diagonal matrix, the first Newton iteration $\mathbf{V}^{1}$ already gives the exact solution of Eq. (16):

$$
\mathbf{V}^{1}=\left(\mathbf{M}+\mathbf{A} \mathbf{G}_{m}\right)^{-1} \mathbf{A} \mathbf{G}_{m} \mathbf{V}^{0} .
$$


In general, in the vicinity of the resting potential, $\partial I / \partial V_{m}$, which represents the membrane conductance, is positive. In this case, $\mathbf{J}^{n}$ is symmetric positive definite and Eq. (17) is guaranteed to have a unique solution. The linear system (17) was solved using a conjugate gradient method with an incomplete Cholesky preconditioner [7].

\subsection{Validation}

In order to illustrate the efficiency of the method as well as its accuracy, the steady-state membrane potential profile was computed in two test cases.

The first test case was a cable (discretization: $100 \mu \mathrm{m}$; see Fig. 1A) comprising 100 myocytes (Courtemanche et al. model) and 10 fibroblasts (MacCannell et al. model) in a configuration similar to that in [8]. The coupling conductance was set to $1 \mu \mathrm{S}$, except at the junction between the myocytes and fibroblasts, where the coupling was $0.1 \mu \mathrm{S}$.

The second test case was a two-dimensional microstructure model similar to that proposed by Spach et al. [9]. The tissue model, 1.73 by $1.73 \mathrm{~mm}$ in size with a discretization of $8 \mu \mathrm{m}$, contained 864 randomly-shaped myocytes [10]. The tissue architecture is shown in Fig. 2A. To approximate the gap junction distribution experimentally observed in adult cardiac tissues, $70 \%$ of the lateral connections were randomly removed [10]. The conductance of the remaining connections was set to $0.2 \mu \mathrm{S}$. The resistivity of the intracellular medium was set to $150 \Omega \mathrm{cm}$. Clusters of fibroblasts covering about $11 \%$ of the tissue were introduced (Fig. 2A). Each 8 by $8 \mu$ m element in these regions was assumed to be a fibroblast with a capacitance of $6.3 \mathrm{pF}$. The conductance of both the fibroblast-myocyte and the fibroblast-fibroblast connections were set to $3 \mathrm{pS}$. The membrane kinetics used was the Ramirez et al. model for the myocytes [11] and the MacCannell et al. model for the fibroblasts [2].

\section{Results}

Figure 1A shows the steady-state membrane potential profile for the first test case. This profile is in agreement with that obtained in [8] by simulating a free evolution until the steady-state was reached. However, because of the slow time constants involved both in the fibroblast model and in the myocyte model, as well as the small fibroblast-myocyte coupling conductance, this technique requires simulating the system for a very long time. Figure 1B compares these methods. While a few iterations were sufficient to reach the steady state with the method presented in this paper, the convergence was much slower when simulating free evolution. In the latter case, an accuracy of $0.2 \mathrm{mV}$ was reach after $100 \mathrm{~ms}$ of simulated time, but $60 \mathrm{~s}$ of simulated time was necessary to obtain and accuracy of $10^{-3} \mathrm{mV}$. Note that the two methods agree
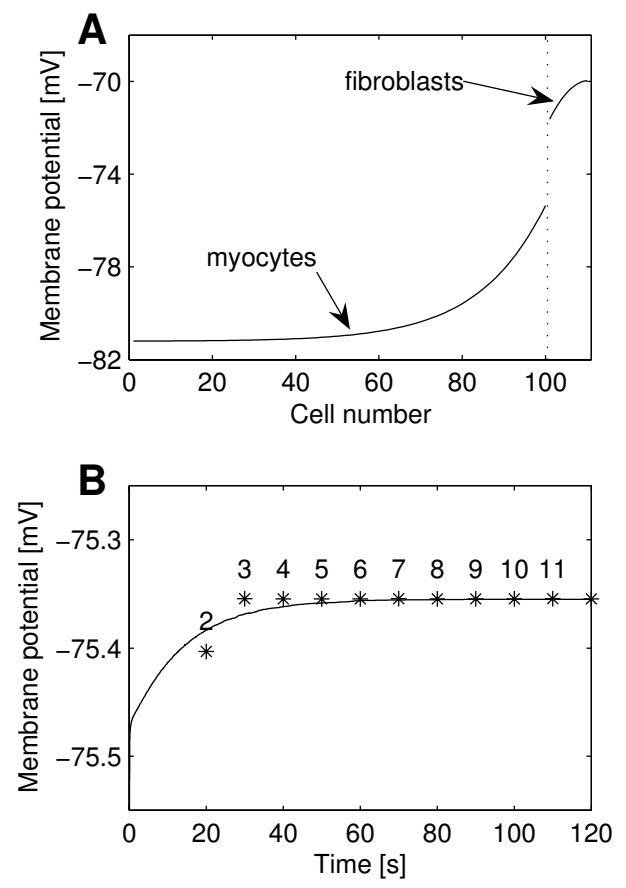

Figure 1. (A) Steady-state membrane potential profile the first test case (cable). (B) Solid line: membrane potential of myocyte \#100 during free evolution starting from its intrinsic resting potential. Stars: successive iterations using the method of this paper (iteration number above the symbol). At iteration 1 , the potential is $-72.9 \mathrm{mV}$ (not shown).

within $5 \cdot 10^{-4} \mathrm{mV}$.

The steady-state membrane potential map for the second test case is displayed in Fig. 2B. As a result of the coupling with fibroblasts, the myocyte membrane potential was non-uniformly depolarized by $3.9 \mathrm{mV}$ up to more than $6 \mathrm{mV}$ close to large clusters of fibroblasts (the intrinsic resting resting potential of the Ramirez model is $-83.7 \mathrm{mV})$. Convergence was reached after 36 iterations. The accuracy of the solution was determined by simulating a free evolution starting from the steady state computed using the non-linear solver. The maximum variation in membrane potential during $200 \mathrm{~ms}$ simulation was $0.42 \pm 0.03 \mu \mathrm{V}$ (max: $0.62 \mu \mathrm{V}$ ). The accuracy of the conjugated gradient solver was found to be more critical for the non-linear solver than for the semi-implicit monodomain simulation. Thus, a smaller relative tolerance was used $\left(10^{-8}\right.$ instead of $\left.10^{-6}\right)$. In any case, the root finding procedure was less demanding in terms of CPU time than simulating the system for $10 \mathrm{~ms}$ using a semi-implicit scheme. These $10 \mathrm{~ms}$ of simulation would be clearly insufficient reach the steady state (see Fig. 1B). 

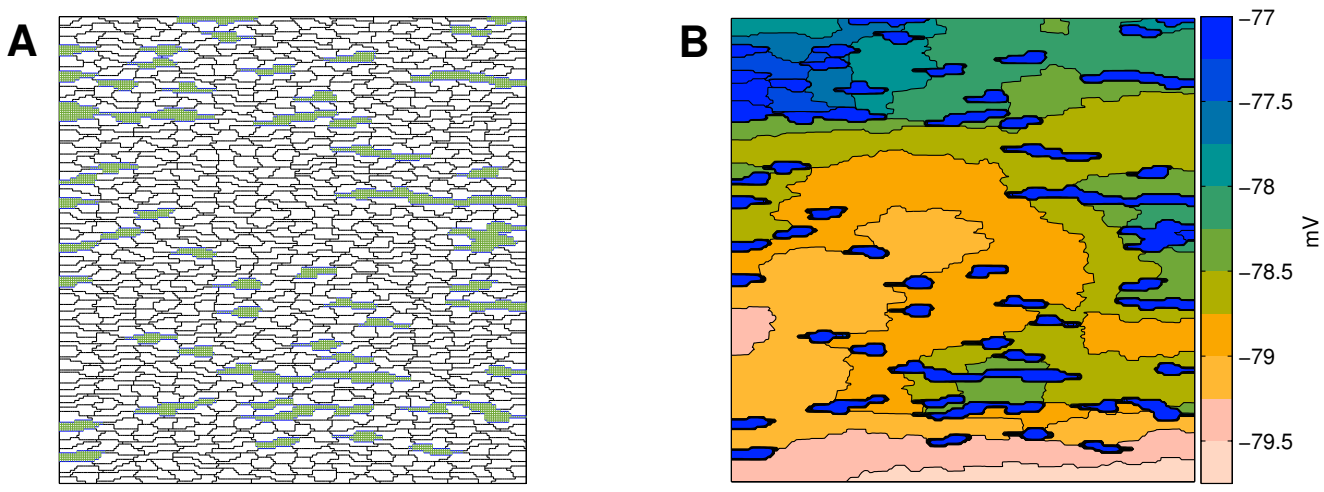

Figure 2. (A) Microstructural model, 1.73 by $1.73 \mathrm{~mm}$ in size, highlighting cell boundaries. Shaded regions represent clusters of fibroblasts. (B) Steady-state myocyte membrane potential map. Isopotential lines are drawn every $0.25 \mathrm{mV}$.

\section{Discussion and conclusions}

Computing of the steady state through root-finding was found to be convenient and computationally efficient when applied to heterogeneous tissue incorporating multiple membrane kinetics models. Generalization to other geometries and to three dimensions is straightforward. Another key advantage is that the convergence properties only depends of $I\left(V_{m}\right)$ and not on the kinetics of the internal variables q. If $I\left(V_{m}\right)$ is nearly linear close to its resting state (which is the case in most membrane models), an accurate estimate is obtained after a few iterations only. However, the algorithm and its efficiency rely on the ability to accurately compute the function $\mathbf{G}\left(V_{m}\right)$ for each of the membrane models involved, which requires some preliminary (handwritten or with the help of a computer algebra system) mathematical computations [3].

\section{Acknowledgements}

This work was supported by the Swiss National Science Foundation (SNSF grant PA002-113171) and NIH grant R01HL76767. The authors thank Marjorie-Letitia Hubbard for providing the microstructural mesh for the second test case.

\section{References}

[1] Kneller J, Ramirez RJ, Chartier D, Courtemanche M, Nattel $\mathrm{S}$. Time-dependent transients in an ionically based mathematical model of the canine atrial action potential. Am J Physiol Heart Circ Physiol 2002;282:H1437-51.

[2] MacCannell KA, Bazzazi H, Chilton L, Shibukawa Y, Clark RB, Giles WR. A mathematical model of electrotonic inter- actions between ventricular myocytes and fibroblasts. Biophys J 2007;92:4121-32.

[3] Jacquemet V. Steady-state solutions in mathematical models of atrial cell electrophysiology and their stability. Math Biosci 2007;208:241-69.

[4] Plonsey R, Barr RC. Bioelectricity: A Quantitative Approach. Second edition. Kluwer Academic Plenum Publishers, 2000.

[5] Luo CH, Rudy Y. A model of the ventricular cardiac action potential June 1991;68(6):1501-1526.

[6] Corless RM, Gonnet GH, Hare DEG, Jeffrey DJ, Knuth DE. On the Lambert W function. Adv Comput Math 1996; 5:329-59.

[7] Golub GH, Van Loan CF. Matrix computations. Johns Hopkins University Press, 1996.

[8] Jacquemet V. Pacemaker activity resulting from the coupling with nonexcitable cells. Phys Rev E 2006;74:011908.

[9] Spach MS, Heidlage JF, Dolber PC, Barr RC. Mechanism of origin of conduction disturbances in aging human atrial. Heart Rhythm 2007;4:175-85.

[10] Hubbard ML, Henriquez CS. Role of cross-cell coupling on impulse propagation in cardiac tissue: A model study. Europace 2007; (in press).

[11] Ramirez RJ, Nattel S, Courtemanche M. Mathematical analysis of canine atrial action potentials: rate, regional factors, and electrical remodeling. Am J Physiol Heart Circ Physiol 2000;279:H1767-85.

Address for correspondence:

Vincent Jacquemet

136 Hudson Hall, Box 90281

Durham, NC 27708, USA

vincent.jacquemet@a3.epfl.ch 